\title{
May thermal imaging be useful in cyclist endurance tests?
}

\author{
Armand Cholewka ${ }^{1}$ Teresa Kasprzyk ${ }^{1} \cdot$ Agata Stanek $^{2}$. \\ Karolina Sieroń-Stołtny ${ }^{3} \cdot$ Zofia Drzazga $^{1}$
}

Received: 21 October 2014/Accepted: 18 March 2015/Published online: 9 April 2015

(C) The Author(s) 2015. This article is published with open access at Springerlink.com

\begin{abstract}
Thermovision is a completely non-invasive and easy-to-perform method of imaging, and it is becoming useful not only in different modalities of clinical medicine, but also in sports medicine. However, it seems that thermal imaging may also find some use in training. That is why the aim of these studies is to compare temperature parameters with data obtained from specialist equipment, which uses monitoring to choose organism parameters during the endurance test and also to determine the efficiency level. In this study, we also try to estimate body metabolism, using a modified formula of Kleiber's law. We describe how metabolism changes during efficiency tests and compare these changes with parameters checked by an ergospirometer. All studies were performed by thermovision camera (Flir Systems E60). Results of the study showed that thermal imaging may be useful in sports medicine as a helpful method in efficiency evaluation. We obtained a high correlation between the standard parameters used in efficiency tests and the parameters derived from thermal imaging, which may suggest the possibility of using thermovision as an additional method in the planning of training cycles, and its effects.
\end{abstract}

Armand Cholewka

armand.cholewka@gmail.com

1 Department of Medical Physics, A. Chełkowski Institute of Physics, University of Silesia, Uniwersytecka 4, 40-007 Katowice, Poland

2 Chair and Clinic of Internal Diseases, Angiology and Physical Medicine, Medical University of Silesia, Batorego 15, 41-902 Bytom, Poland

3 Department of Physiotherapy, School of Health Sciences, Institute of Physical Medicine, Medical University of Silesia, Medyków 12, 40-752 Katowice, Poland
Keywords Thermal imaging in sport - Organism metabolism · Progressive tests - Body temperature

\section{Introduction}

In recent years, applications of thermography have become more and more popular. Progress can be observed in many different areas of medicine and science [1-8]. As a completely non-invasive and fast method, thermovision has become useful in sport [9-14]. A few decades ago, the basic aim of athletes was to study definitive increases or decreases in skin temperature during specified periods of exercise (running, cycling or swimming), and also the diagnostics of injuries which led to changes in body surface temperature [1,8-12]. Nowadays, it is known that thermal imaging can give important information about the state of health of the sportsman as well as give some information about many different diseases and methods of recovery after different injuries $[9,15,16]$.

There are possibilities to evaluate changes in some physical parameters, like the stream of heat energy radiated from the body or the metabolism changes during sports activity using thermal parameters derived from thermal images. However, if the body surface temperature is known, it could be possible to estimate the average temperature of the whole body by using the experimental law as in Eq. (1) [14, 17]:

$$
\begin{aligned}
T_{\text {body }}= & 0.07 T_{\text {foot }}+0.32 T_{\text {tibia }}+0.17 T_{\text {back }}+0.18 T_{\text {chest }} \\
& +0.14 T_{\text {arms }}+0.05 T_{\text {hands }}+0.07 T_{\text {head }}
\end{aligned}
$$

$T_{\text {foot }}$, foot surface temperature; $T_{\text {tibia }}$, calf surface temperature; $T_{\text {back }}$, back surface temperature; $T_{\text {chest }}$, chest surface temperature; $T_{\text {arms }}$, arms surface temperature; 


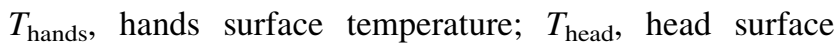
temperature.

The empirical Eq. (1) is based on measurements of surface temperatures of individual body parts. It can be seen that some parts of the body (tibia, chest, arms and back) have a bigger influence on the average whole body temperature which makes such estimations easier using thermovision in sports diagnostics, especially in the cyclist endurance test which is the goal of this work [17].

Additionally, it is possible to estimate the power of radiation loss from the surface of the body during sports activities using the Stefan-Boltzmann law [4, 14, 18, 19]:

$\Phi_{R}=\sigma e A\left(T_{\text {body }}^{4}-T_{\text {enviroment }}^{4}\right)$

where $\sigma$, Stefan-Boltzmann constant, equal to $5.67 \times 10^{-8}\left[\mathrm{Wm}^{-2} \mathrm{~K}^{-4}\right] ; e$, radiation surface emissivity; $A$, body surface.

The stated effort tests (so-called progressive tests) are based on a regular increase in exercise intensity. These tests are usually performed on a bicycle ergometer with a gauge to measure pedal power. The reaction of the cardiovascular system on an increase in sports training can be specified by efficiency tests. Moreover, such tests make it possible to define the anaerobic threshold related to the athlete's pulse [20, 21].

The aim of the study was to compare temperature parameters with data from specialist equipment, which is used to monitor chosen organism parameters during the endurance test and also to determine the efficiency level.

\section{Materials and methods}

Twelve male cyclists participated in the study. During the research, the subjects were healthy, without any coexisting illnesses. All studies were performed in the Sport Diagnostic Centres Diagnostix in Katowice and Wisła, Poland, which provide services regarding efficiency tests for athletes. All of the tested cyclists were at an intermediate level of cycling skill. The subjects came from various sports teams, and half of them were competitors in Banimex MTB Team Dabrowa Górnicza.

The measurements were taken during standard sportsmen's efficiency tests. The research included standard training cycles for each of the athletes.

Sportsmen were wearing ergospirometry masks (Cortex Metamax 3b, the specialist telemetry system CPET "BREATH-BY-BREATH") that are needed to measure parameters like $\mathrm{VO}_{2}$ (the amount of intake oxygen) and $\mathrm{VE}$ (ventilation per minute, which is the volume of air flowing through the lungs per $1 \mathrm{~min}$ ). The researchers used spirometric parameters to measure the correlations between the temperature of the body surface, body core and metabolism, which were possible to count through the thermal parameters obtained from the curves.

Moreover, during the efficiency tests, blood samples were taken every 3 min from a finger from every sportsman. The lactate level was calculated from these blood samples.

To carry out the tests, a thermal imaging camera was used (Flir Systems E60 with sensitivity $0.05 \mathrm{~K}$ ). Each of subjects had to go through a process to adapt their body temperature to the environment, which takes about 30-40 min. The temperature of the environment in test room was monitored and kept at a stable level $\left(21 \pm 1{ }^{\circ} \mathrm{C}\right)$. The examined area of the body remained exposed.

According to the empirical formula (1), which offers the possibility to estimate the whole body surface based on measurements of surface temperatures of individual body parts, it was possible to estimate body temperature changes during the efficiency tests. It can be seen from this formula (1) that some parts of the body (tibia, chest, arms and back) have a bigger influence on the whole body average temperature, so it was possible to use this equation in the cyclist endurance tests, which is the goal of this work. During the efficiency test, researchers measured the surface temperature of the sportsmen's faces [instead of the head in formula (1)], chest, arms, back and calf [measured instead of the tibia in formula (1)]. We replaced the tibia surface temperature with the calf because of limited access to measure the fronts of legs. The exclusion of the tibia from measurement due to the legs being in motion came about because of the dynamic changes in the angle between the tibia surface and the thermal camera.

The curves were performed in 3-min intervals, which allowed us to get more exact results. Some measurements were difficult to perform, i.e. the movement of the limbs and the leaning position of the body created some difficulties. In these cases, it was necessary to record an infrared movie and choose the best curves for analysis. Researchers also tried to set the camera perpendicular to better capture the surface of the body.

However, all the measurements were performed in a similar way to the Glamorgan Protocol, which included thermal imaging in its medical standards and rules [22, 23].

Consequently, the researcher had to adapt to the conditions with which the athletes found themselves during the efficiency test. It is important not to disturb the cyclist during the effort test and try to provide as much comfort as possible.

The bikes were installed on a special device that provides power measurement. Also, an ergospirometry mask was worn by each cyclist. After these preconditions, the effort test was started. All efficiency tests started from $50-\mathrm{W}$ threshold which increased every $3 \mathrm{~min}$ by $30 \mathrm{~W}$. The 
test ended when the cyclist could not continue his effort due to reaching maximum exertion. Every athlete had a maximum exertion level when participant could not continue the effort. Every test took about $30 \mathrm{~min}$.

The curves were analysed by using a ThermaCAM TM Researcher Pro 2.8 SR-3. Statistical analyses were done in Statistica 10.

\section{Results and discussion}

The goal of this work was to compare thermal parameters calculated from curves of the body surface during efficiency tests with the parameters from special devices normally used in such tests like cycloergometer power measurements or blood research.

Figure 1 presents curves of representative cyclists' body parts performed at the beginning (a), after $6 \mathrm{~min}$ (b), $12 \mathrm{~min}$ (c), $18 \mathrm{~min}$ (d), $24 \mathrm{~min}$ (e) and $30 \mathrm{~min}$ (f) of testing.

Changes in body surface temperature during training are visible. It can be easily seen on the curves presented in Fig. 1. We reduced regions of interest to those mentioned in the methodology, due to the impossibility to measure feet and hands during such exercises. The curves of faces were done excluding areas covered by the ergospirometry mask. However, all regions that were taken into account in the estimation of the whole body average temperature were the biggest contributors to the average of body surface temperature.

The average athletes' body surface temperature was calculated based on Eq. (1), and the results were graphed (Fig. 2).

The internal body temperature and its changes were also measured during the effort tests. The participants were captured by a thermal camera from the corner of the eye, and the results are presented in Fig. 3 and also in Table 1.

One can see from Figs. 2 and 3 that the average body surface temperature changes and the body core temperature does not change linearly during the test time. We can see a decrease at the beginning of the test which is seen until the 12-min mark, and next some oscillations of average body surface temperature were observed. Such a result may be correlated with the fact that the body is sweating, whereas changes in the core temperature seem to take shape resembling a sigmoidal curve. This kind of body core temperature change probably is related to thermoregulation mechanisms which are activated during sports training. We can see that the body reacts by a change in the core temperature after some time. It should be noticed that the core temperature rises irregularly during the effort test. After $30 \mathrm{~min}$, the average core temperature increased from 34.9 to $35.9{ }^{\circ} \mathrm{C}$. The increase in the core temperature due to the regular rising of sport activity load seems to be an expected situation. We might suppose that the cyclist's body has cooled down easier at the beginning of training because at the beginning of the test, the thermoregulation system is more efficient due to a smaller load. As we can find in sport literature, the rate of heat transfer from the body core to skin depends from the gradient between the body core and the body surface temperature. During the sport effort, the temperature gradient rising up is because of acceleration of metabolism [17].

It can be seen that the average core body temperature plot presented in Fig. 3 reveals two regions of quite stabilised temperature-between 5 and 12 as well as between 21 and 28 min of training. After the 12th minute of training, the effort is so intense that the core temperature rises much more dynamically and almost linearly. Simultaneously, the body surface temperature decreases in the 15th minute, and after that the temperature stabilises. The decrease in body surface temperature at the beginning of the effort test may be connected with the movement of blood in the cutaneous vasculature to the muscles, where the work is done and oxygen is needed. In point of fact, blood circulation is very important in heat transport of the body. On the other hand, heat exchange on the surface of our bodies will not occur immediately due to the time needed for microcirculation changes in the capillaries, which are responsible for skin temperature. Both processes are dependent on one another and heat transport will take some time, which is a consequence of body core thermoregulation inertia. The mechanism of heat exchange is activated when information about body and blood temperature change is delivered to the hypothalamus. The activation of the eccrine sweat gland causes sweat to be secreted onto the skin surface, thereby promoting the process of heat loss activation. These activations of thermoregulation mechanisms start after some time needed for the body to react to the stimulus. However, the thermoregulation centre is active until the equilibrium state is reached [17]. The aforementioned processes and their results are easily seen in thermal imaging as well as in the parameters derived from curves (presented in Figs. 1-3).

The question was raised-is it possible to use only thermal imaging to evaluate the energy and power of sportsmen? Normally such parameters are calculated from the apparatus used in progressive tests. That is why correlations between average power from a bicycle ergometer and average body surface temperature as well as average power radiation of athletes' bodies during tests were calculated. Actually, the power from a bicycle ergometer is calculated from load applied on bike. The load increased until the athlete discontinued the effort. This is the standard procedure of efficiency test, which we can find in others sport publications. 
(a)
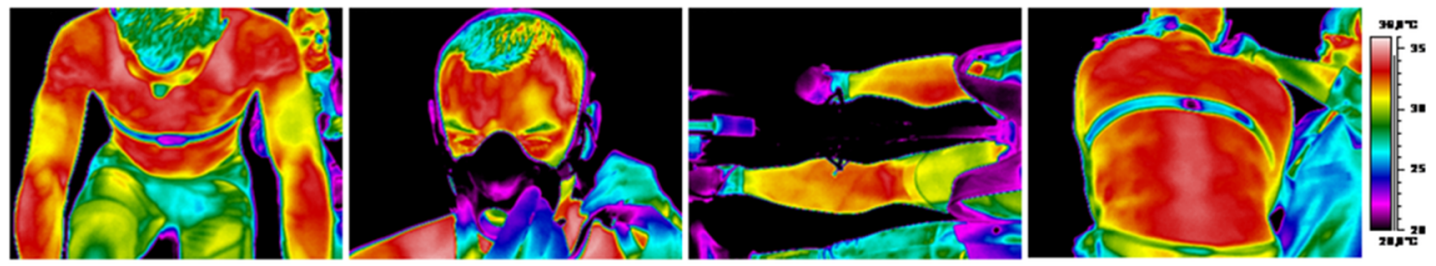

(b)
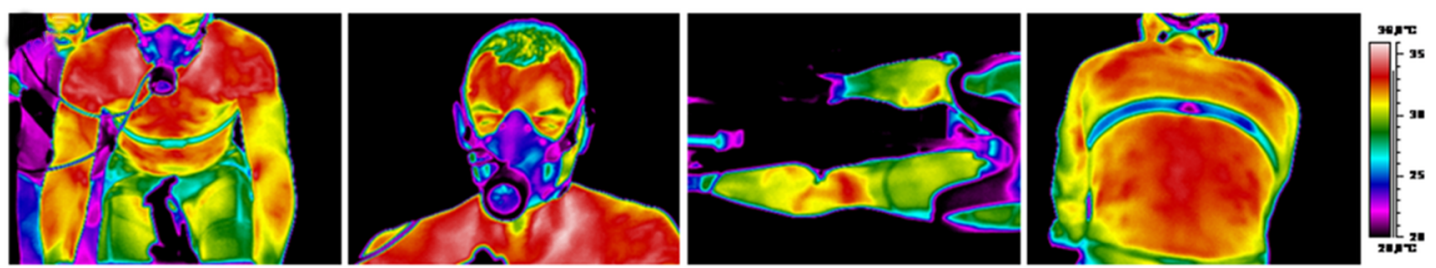

(c)
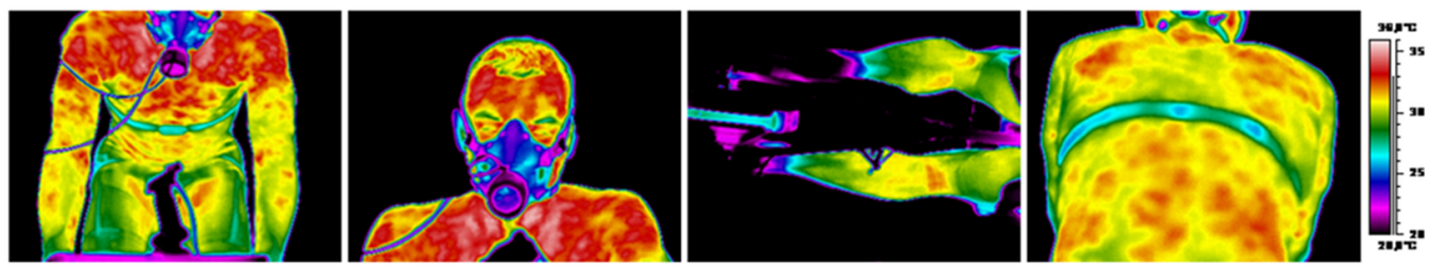

(d)
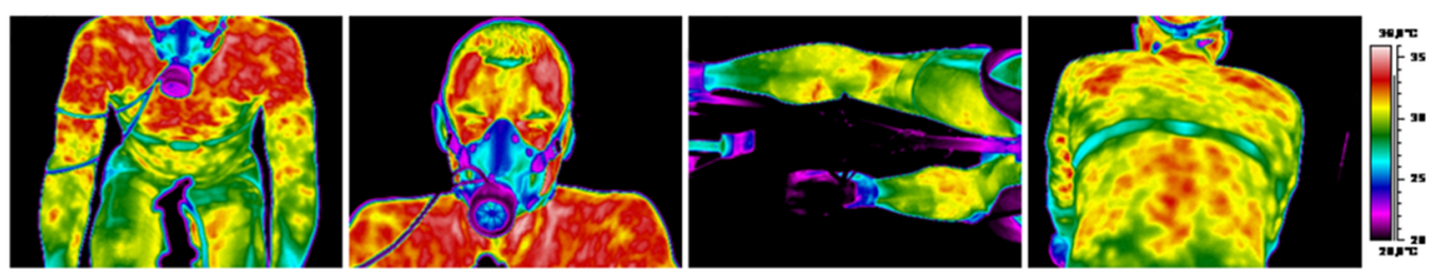

(e)
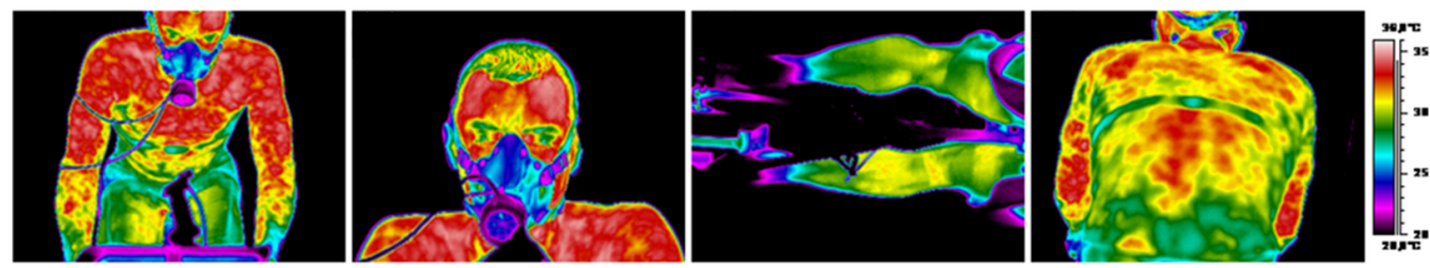

(f)
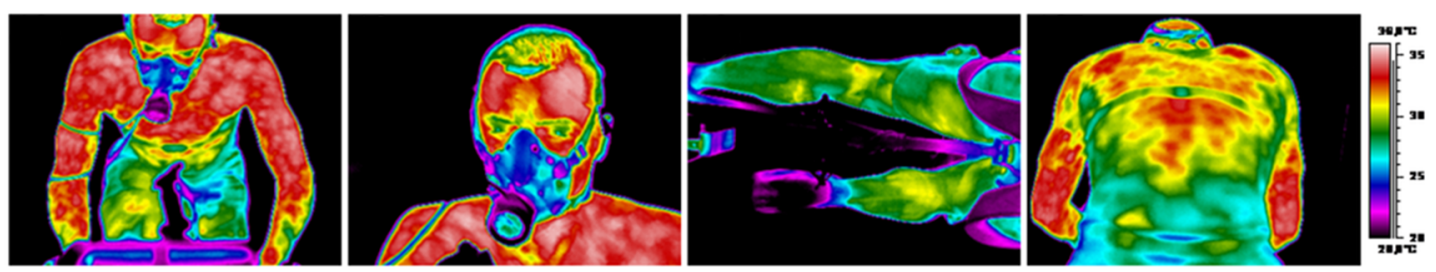

Fig. 1 Curves of representative cyclists' body parts performed at the beginning (a), after $6 \mathrm{~min}(\mathbf{b}), 12 \mathrm{~min}(\mathbf{c}), 18 \mathrm{~min}(\mathbf{d}), 24 \mathrm{~min}(\mathbf{e})$ and $30 \mathrm{~min}(\mathbf{f})$ of test

We obtained a high value of negative correlation coefficient $(r=-0.70)$ between average power displayed on the bicycle ergometer and average body surface temperature counted from thermal parameters, showing that body surface temperature changes are strongly dependent on power produced by the human body. This dependency was presented in Fig. 4. The negative dependence seems to be the effect of switching on the thermoregulation processes and the cooling of the body due to sweating. In addition, hard work is performed by the cyclist and we could expect an increase in inner body temperature, because the effort leads to increased body metabolism and also increased core temperature. However, interesting results seem to be the correlation between ergometer power and average radiated power from the human body calculated basically in Eq. 2 (Fig. 5). In this case, the obtained correlation factor was also very high $(r=0.89)$ but positive.

According to recently gained knowledge of authors, such studies were not provided and there is lack of other similar data.

Huge potential for thermal imaging in sports as a diagnostic method and also a non-invasive technique that can 


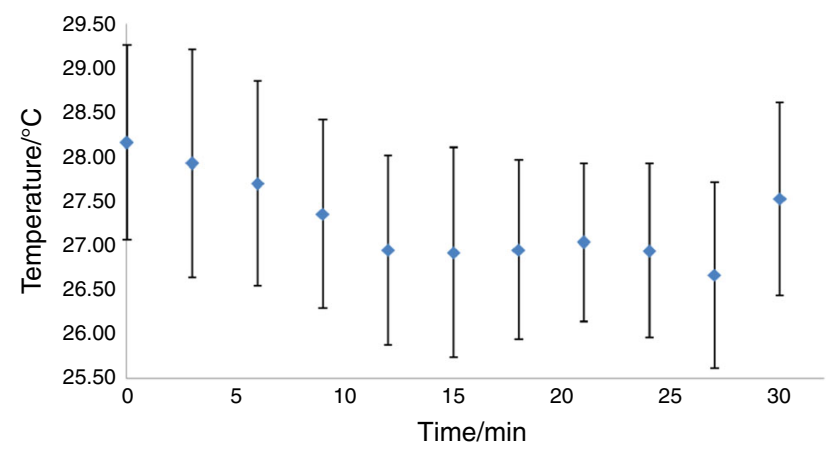

Fig. 2 Changes in average body surface temperature (Temperature $\left.\left[{ }^{\circ} \mathrm{C}\right]\right)$ obtained for the whole group of participants based on Eq. (1) depending on the time results of the effort test

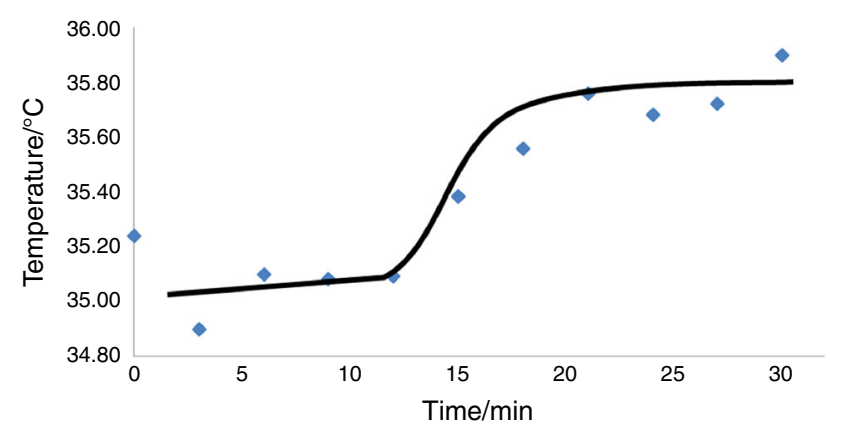

Fig. 3 Changes in average body core temperature $\left[{ }^{\circ} \mathrm{C}\right]$ calculated from the corner of the eye from facial thermal images

Table 1 Average internal temperature of the athlete's body calculated from the eye corner

\begin{tabular}{ll}
\hline Effort time/min & Body core temperature $/{ }^{\circ} \mathrm{C}$ \\
\hline 0 & 35.24 \\
3 & 34.90 \\
6 & 35.10 \\
9 & 35.08 \\
12 & 35.09 \\
15 & 35.38 \\
18 & 35.56 \\
21 & 35.76 \\
24 & 35.68 \\
27 & 35.72 \\
30 & 35.90 \\
\hline
\end{tabular}

be used in the analysis of temperature as well as energy produced by a cyclist was confirmed by very high correlation coefficients (statistical significance level $p<0.05$ ) obtained for ergometer power and power loss of radiation from the body and for average body surface temperature.

As we consider heat emitted from athletes' bodies during training and effort test standard parameters in this study, it might be justified to consider convection and

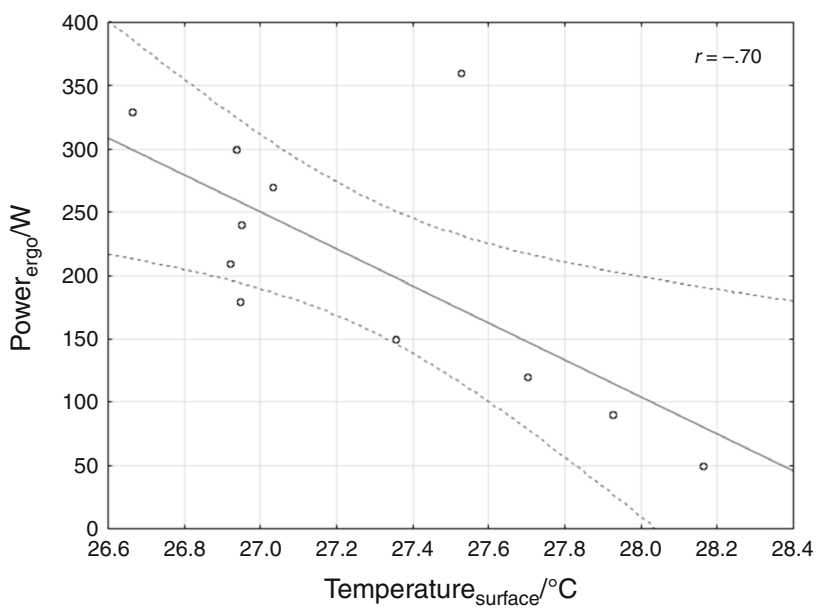

Fig. 4 Correlation between power set on bicycle ergometer Power ergo [W] and average body surface temperature of athletes during progressive tests

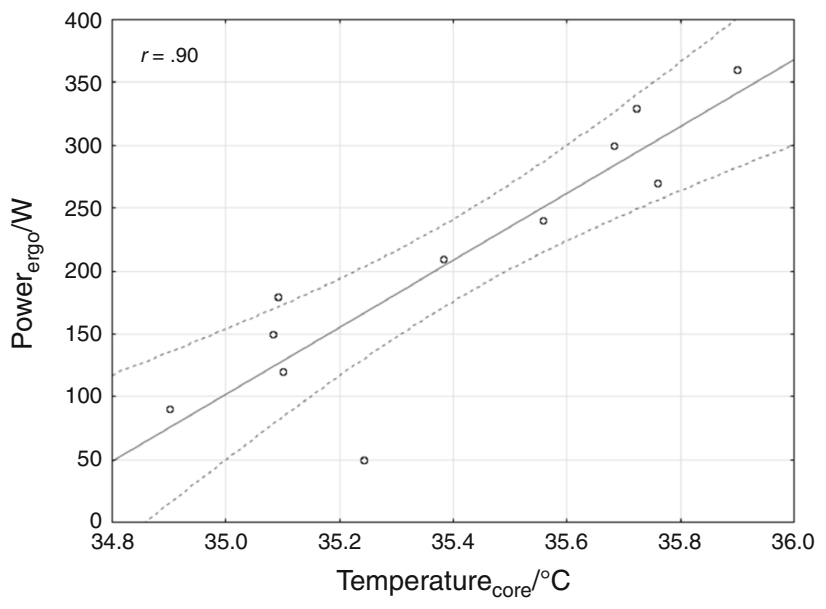

Fig. 5 Correlation between power set on bicycle ergometer Power $_{\text {ergo }}$ [W] and power loss of radiation counted according to Eq. 2 obtained from group of athletes during progressive tests

conduction heat loss in energy expenditure in the calculations. However, it seems that for such correlations, it is easier and sufficient to use only the estimation of power loss of radiation due to the biggest contribution of power loss of radiation in the heat transfer of the organism.

There are also other very important parameters analysed during efficiency tests of sportsmen. One of them is $\mathrm{V}_{\mathrm{O}_{2} \max }$ which identifies physical activity levels and indicates any disorders in the cardiovascular system. The oxygen capacity of the body and metabolised oxygen amount are showed by the $\mathrm{V}_{\mathrm{O}_{2} \max }$ parameter. By using the ergospirometry mask on athlete's face, measurement of $\mathrm{V}_{\mathrm{O}_{2} \max }$ parameter is done during training. A special sensor is installed on the mask and we can measure the oxygen consumption $\mathrm{V}_{\mathrm{O}_{2}}$ and carbon dioxide secretion $\mathrm{V}_{\mathrm{CO}_{2}}$. Maximum oxygen consumption $\mathrm{V}_{\mathrm{O}_{2} \max }$ occurs when the 


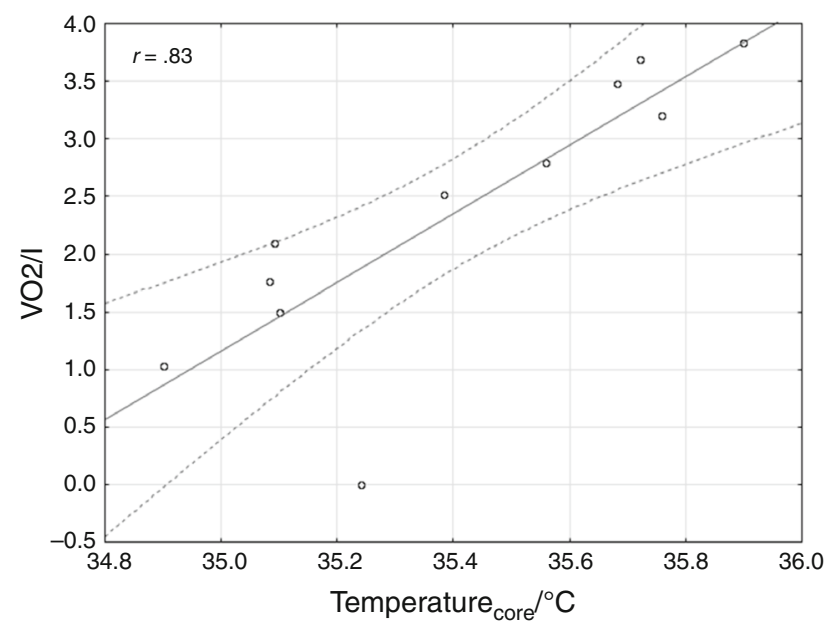

Fig. 6 Correlation between oxygen capacity of the body and metabolised oxygen which is described by $\mathrm{V}_{\mathrm{O}_{2} \max }[1]$ and temperature core derived from the thermal images

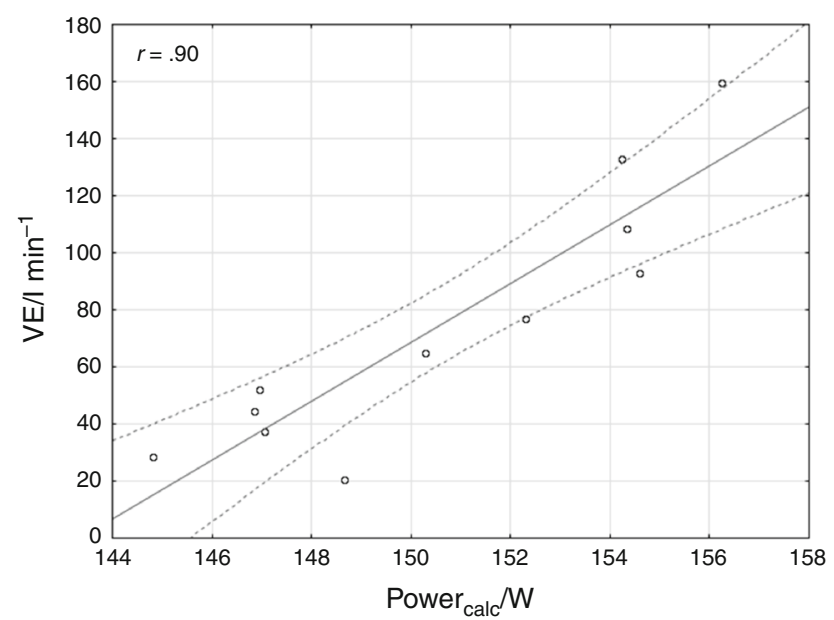

Fig. 7 Correlation between ventilation VE $\left[1 \mathrm{~min}^{-1}\right]$ parameter and radiation power loss from cyclist body $P_{\text {calc }}$

$\mathrm{V}_{\mathrm{O}_{2}}$ parameter relative to the increased load achieves a plateau stage [1]. The oxygen consumption-to-carbon dioxide secretion ratio is defined as a respiratory exchange ratio. The other very important parameter is ventilation per minute (VE) which is defined as a volume of oxygen flowing through the lungs per minute. Both parameters are important forms from an athletes' efficiency point of view. That is why another question was raised-if it is possible to estimate the parameters just using the body temperature derived from thermal imaging.

For deeper insight into the problem, correlations between oxygen capacity of the body and metabolised oxygen, which are described by $\mathrm{V}_{\mathrm{O}_{2} \max }$, and temperature core were performed. Moreover, correlations between the ventilation per minute (VE) parameter and the power loss of radiation from the athlete's body were calculated according to Eq. 2. The results obtained are shown in Figs. 6 and 7, respectively.

As we can see in Figs. 6 and 7, correlations coefficients are very high and theirs values were $r=0.83$ for oxygen capacity of the body and temperature core and $r=0.90$ in the case of correlation between ventilation parameter and power loss of radiation from cyclist body. For it was confirmed by statistical significance factor $p<0.05$.

Such high and statistically significant values of the correlation ratios for the covered parameters indicate a possibility to estimate them based on thermal imaging measurements, which might prove quite inexpensive and fast in performance. Also, it is a completely non-invasive technique, so athletes might focus only on effort during their tests. Nowadays, it is necessary to take blood samples every $3 \mathrm{~min}$ which can distract the athletes. In the case of thermal imaging, sportsmen may not even be aware that the study is in progress. Perhaps the progress of thermal imaging in sport may lead to the use of thermal imaging not only for monitoring of health but also for endurance tests and metabolic changes during sports training. Thermovision may also provide useful information for planning and training schedules for player.

\section{Conclusions}

The results obtained from our studies show that thermal imaging may be useful in sports not only as a diagnostic tool, but may be additionally as a method helpful for athletic efficiency evaluation.

A high correlation between standard parameters used in efficiency tests and parameters derived and calculated from thermal imaging may suggest possibilities for the use of thermovision as a complementary addition to some standard methods used in the planning of training cycles. It suggests that the thermovision method could be a viable support to standard ergospirometry, and it might offer some additional parameters.

Open Access This article is distributed under the terms of the Creative Commons Attribution 4.0 International License (http:// creativecommons.org/licenses/by/4.0/), which permits unrestricted use, distribution, and reproduction in any medium, provided you give appropriate credit to the original author(s) and the source, provide a link to the Creative Commons license, and indicate if changes were made.

\section{References}

1. Coh M, Sirok B. Use of the thermovision method in sport training. Facta Univ Phys Educ Sport. 2007;5(1):85-94.

2. Mikulska D, Maleszka R. Use of thermography study as a diagnostics metod in dermtology. Clin Dermatol. 2002;4:57-60. 
3. Nowakowski A. Progress in thermography-medical applications. Gdańsk: Wydawnictwo Gdanskie; 2001.

4. Cholewka A, Drzazga Z, Kajewski B, Bogucki R, Wiśniowska B. Thermal imaging of skin body surface due to whole body cryotherapy—preliminary report. Phys Med. 2004;1:81-3.

5. Cholewka A, Stanek A, et al. Thermography study of skin response due to whole-body cryotherapy. Skin Res Technol. 2011;18:180-7.

6. Cholewka A, Drzazga Z. Thermovision diagnostics in chosen spine diseases treated by whole body cryotherapy. J Therm Anal Calorim. 2010;102:113-9.

7. Ring JF, Mercer J. Thermal imaging for fever screening. Mag Int Org Stand. 2007;4:33-5.

8. Clark RP, Mullan BJ, et al. Skin temperature during running-a study using infrared colour thermography. J Physiol. 1977;267:53-62.

9. Keyl W, Lenhart P. Thermography in sport injuries and lesions of the locomotor system due to sport. Fortschr Med. 1975;93(3): 124-6.

10. Wade CE, Veghte JH. Thermographic evaluation of the relative heat loss by area in man after swimming. Aviat Space Environ Med. 1977;48(1):16-8.

11. Veghte JH, Adams WC. Temperature changes during exercise measured by thermography. Aviat Space Environ Med. 1979;50(7):708-13.

12. Nakayama T, Ohnuki Y, et al. Fall in skin temperature during exercise observed by thermography. Jpn J Physiol. 1981;31(5): $757-62$.
13. Cholewka A, Stanek A, Kasprzyk T. Thermovision application in sport efficiency test-pilot study. PAK. 2013;59:871-4.

14. Cholewka A. The influence of low temperature on organism, in vitro and in vivo studies. A. Chełkowski Institute of Physics, University of Silesia Katowice Poland, Doctor Thesis; 2005.

15. Bauer J, Hurnik P, Zdziarski J, Mielczarek W, Podbielska H. Thermovision and its applications in medicine. Acta Bio-Optica Inform Med. 1997;3(2-4):121-31.

16. Maksymowicz K, Dudek K, Bauer J, Jurek T, Drozd R. Asessment of the possibilities of applications of the thermovision technique in medico-legal diagnosis. Ann Acad Med Stetin. 2007;53(Suppl. 2):102-6.

17. Pilawski A. Biophysics basics. Warszawa: PZWL; 1983.

18. Tuszynski J, Dixon J. Biomedical applications for introductory physics. New York: Wiley; 2002.

19. Wiśniewski S, Wiśniewski ST. Heat transfer. 6th ed. Warszawa: WNT; 2009, ISBN 978-83-204-3671-6.

20. Friel J. Bible of mountains cyclist trening. Zielonka: Buk Rower; 2004.

21. Zatoń M, Hebisz R. Physiologist basics of mountains biking trening. Wrocław: AWF Wrocław; 2011.

22. Ammer K. The Glamorgan Protocol for recording and evaluation of thermal images of the human body. Thermol Int. 2008;18(4):125-44.

23. Ring EFJ, Ammer $\mathrm{K}$. The technique of infrared imaging in medicine. Thermol Int. 2000;10(1):7-14. 